FIXED POINT THEOREMS FOR EXPANDING MAPS

by

A. A. G111esple and B. B. W1111ams

Department of Mathematics

The Untversity of Texas at Arlington

Ar1ington, Texas 76019

Techntca1 Report No. 154

March, 1981

UNIVER:

AT 


\section{FIXED POINT THEOREMS FOR EXPANDING MAPS}

\section{A. A. Gillesple and B. B. Willians}

Suppose $(x, d)$ to a metrlc space, $h>0$ and $T: X+X$. We shall use the notation $T \in E(h)$ to mean $d(T(x), T(y)) \geq h d(x, y)$ for each $x, y \in X$. If $h>1$, then $T$ w111 be called an expanding map. c1early $T \in E(h)$ implies $T$ is a 1-1. function and $d\left(T^{-1}(x), T^{-1}(y)\right) \leq \frac{1}{h} d(x, y)$ for each $x, y \in T(X)$.

In this paper some conditions are found to insure that an expanding map w11 have a flxed point. It is shown that each finite dimenatonal Banach space $X$ has the following property: each continuous and expanti map from $x$ into $X$ has a flyed point. It 1.8 also shown that not all Infinte dimenstonal Banach spaces have the above property.

Theorem 1. Suppose that $(X, d)$ is a complete metric space, $T$ Is an expanding map from $X$ into $X$ and $T(X)$ is a closed subset of $X$. The following are equivalents:

(1) Thas a untque flxed potnt.

(11) $m_{n=1}^{n} \tau^{n}(x) \neq \emptyset$.

(11) There extsts $\left[x_{n}\right\}$ a sequence in $x$, such that $d\left(x_{n}, T\left(x_{n}\right)\right)$

Frool. It to clear that (1) Implles (1I) and that if $T$ has a fixed point, then 1 t 18 untque. To see that (11) Implies (111), suppose $p \in \bigcap_{n=1}^{\infty} T^{n}(X)$. Let $F=T^{-1}$ and for each $n \in z^{+} x_{n}=F^{n}(p)$. Now $d\left(x_{n}, T\left(x_{n}\right)\right)=d\left(F^{n}(p), F^{n-1}(p)\right) \leq\left(\frac{1}{h}\right)^{n-1} d(F(p), p) \rightarrow 0$. To see that 
(111) imp11es (1), let $\left\{x_{n}\right\}$ denote a sequence in $x$ such that $d\left(x_{n}, T\left(x_{n}\right)\right)+0$. Now $T \in E(h)$, where $h>1$, and hence $d(T(x), T(y))\left(1-\frac{1}{h}\right)$ $\leq d(T(x), x)+d(T(y), y)$. It follows that $\left\{T\left(x_{n}\right)\right\}$ is a Cauchy sequence In the complete metric space $T(x)$. Thus, there exfsts $T(p)$ such that $T\left(x_{n}\right)+T(p)$. Now $T^{-1}$ is continuous, hence $x_{n} \rightarrow p$, and since $d(T(p), p)$ $\leq d\left(T(p), T\left(x_{n}\right)\right)+d\left(T\left(x_{n}\right), x_{n}\right)+d\left(x_{n}, p\right)$, it follows that $T(p)=p$.

Lemma 1. Suppose (X,d) is a complete metric space, $T$ a function from $X$ into $x$ and $n \in z^{+}$.

(1) $T \in E(h)$ implies $T^{n} \in E\left(h^{n}\right)$.

(11) If $T$ is an expanding map, then $T^{n}(p)=p$ if and only if $T(p)=p$.

(111) If $T \in E(h)$ and $T$ is continuous, then $T(X)$ is complete.

Proof. The truth of (1) is clear. For (11), suppose $h>1$ and $T^{n}(p)=p$. Now $T(p)=T\left(T^{n}(p)\right)=T^{n}(T(p))$, hence $d(T(p), p)$ $=d\left(T^{n}(T(p)), T^{n}(p)\right) \geq h^{n} d(T(p), p)$ which imp11es $T(p)=p$. The truth of (111) follows since $\mathrm{T}^{-1}$ is uniformiy continuous.

It follows from Lemma 1 (111), that in Theorem 1, the requirement that $T(X)$ is a closed subset of $X$ could have been replaced by $T$ is a continun"m map. The following example shows that Theorem 1 no longer holds if $h=1$.

Example 1. Define $T: R^{1} \rightarrow R^{1}$ by $T(x)=x+e^{x}$.

The function $T$ in Example 1 is continuous, both (11) and (111) of Theorem 1 hold and $T \in E(1)$, yet $T$ has no fixed point.

Below we w111 show that if $T: R^{1}+R^{1}$, where $T$ is a continuous and expanding map, then $T$ must have a flxed point. However, there are expanding 
mape from $\mathrm{R}^{1}$ Into $\mathrm{R}^{1}$ with no fixed point.

Example 2. Define $\mathrm{T}: \mathrm{R}^{1}+\mathrm{R}^{1}$ by $\mathrm{T}(\mathrm{x})=3 \mathrm{x}+\mathrm{e}^{\mathrm{x}}$ if $\mathrm{x} \geq 0$ and $T(x)=3 x-e^{x}$ if $x<0$.

The function in Example 2 is an expanding map with no flxed point.

Deflultion. Let $F(h)$ denote the clage of all complete metric spaces $X$ such that if $T: X \rightarrow X$ where $T \in E(h)$ and continuous, then $T$ has $A$ ftxed point.

\section{Theorem 2.}

(1) If $1<h_{1}<h_{2}$, then $E\left(h_{1}\right)=E\left(h_{2}\right)$ (Hence for $h>1$, we

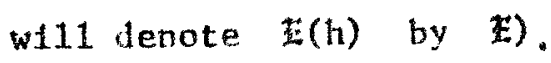

(i1) If $x_{1}$ and $x_{2}$ are Isomorphic Banach spaces, then $x_{1} \in \mathbb{E}$ im $\mathrm{X}_{2} \in \mathrm{z}$

(111) IF $X$ and $Y$ are isometric metric spaces, then $X \in T$ implies $\mathbf{Y} \in \mathbf{X}$

Proor. To move (1), suppose $x \in \mathbb{E}\left(h_{1}\right), T: x+x$, where $T$ is a conctinous and $T \in E\left(h_{2}\right)$. Now $h_{1}<h_{2}$ tmplles $T \in E\left(h_{1}\right)$ thus $T$ has $a$ Exed point and hence $\mathrm{X} \in \mathrm{z}\left(\mathrm{h}_{2}\right)$. Suppose $\mathrm{X} \in \mathrm{X}\left(\mathrm{h}_{2}\right), T: X+X$, where $T$ Is continuous and $T \in E\left(h_{1}\right)$. Thus, by Lemma $1(1)$, there exists $n \in z^{+}$ ench that $\mathrm{b}_{1}^{\mathrm{n}}>\mathrm{h}_{2}$ and hence $\mathrm{T}^{\mathrm{n}} \in \mathrm{E}\left(\mathrm{h}_{2}\right)$. Since $\mathrm{T}^{\mathrm{n}}$ has a fixed point, is

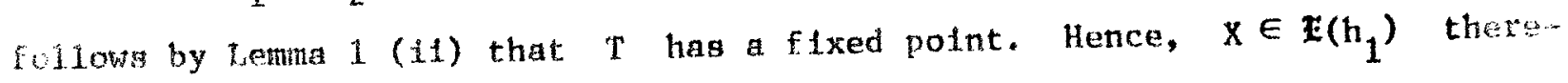
Fore, $\mathbf{E}\left(\mathrm{h}_{1}\right)=\mathbf{E}\left(\mathrm{h}_{2}\right)$.

To gee that (11) is true, suppose that $F$ is a 11near homeomorphism Erom the Benach space $\left(x_{1},\|\|_{1}\right)$ onto the Banach space $\left(x_{2},\|\|_{2}\right), x_{1} \in E$ 
and that $T$ is a continuous and expanding map from $x_{2}$ into $x_{2}$. For some $h>1, T \in E(h)$, thus there exists $n \in z^{+}$such that $1>\left\|F^{-1}\right\| \frac{1}{h^{n}}\|F\|=\frac{1}{k}$. Define $G: X_{1} \rightarrow X_{1}$ by $G=F^{-1}$ o $U$ of where $U=T^{n} \in E\left(h^{n}\right)$. Clearly $G$ is continuous. To see that $G$ is an expanding map, auppose $x, y \in X_{1}$ and let $a=G(x)$ and $b=G(y)$. Now $\|x-y\|_{1}=\left\|G^{-1}(a)-G^{-1}(b)\right\|_{1}=$ $\left\|F^{-1}\left(U^{-1}(F(a))\right)-F^{-1}\left(U^{-1}(F(b))\right)\right\|_{1} \leq\left\|F^{-1}\right\|\left\|U^{-1}(F(a))-U^{-1}(F(b))\right\|_{2} \leq$ $\left\|F^{-1}\right\| \frac{1}{h^{n}}\|F(a)-F(b)\|_{2} \leq\left\|F^{-1}\right\| \frac{1}{h^{n}}\|F\| a-b\left\|_{1}-\frac{1}{k}\right\| G(x)-G(y) \|_{1}$. Thus, $G \in E(k)$ where $k>1$, hence there extsts $p \in X_{1}$ such that $p=G(p)=F^{-1}(U(F(p)))$. Now $T^{n}(F(p))=U(F(p))=F(p)$, thus by Lemma $1, T$ has a fixed point. Therefore, $x_{2} \in \mathbb{x}$.

To see that (111) is true, suppose that $F$ is an isometry from the metric space $(X, d)$ onto the metric space $(Y, p), X \in X$ and that $T$ is a continuous expanding map from $Y$ into $Y$. Now $Y$ is complete, and $G: X+X$ defined by $G=F^{-1}$ o $T \circ F$ is a continuous and expanding map. Since $G$ has a flxed point, it follows that $T$ has a flxed polnt. Therefore, $Y \in Z$. Theorem 3. If $X$ Is a Eintte dimensional Banach space, then $X \in F$. Proof. First suppose $T: R^{n} \rightarrow R^{n}$ where $T$ is a continuous and expandIng map. $T$ IE a homeomorphtsm and from Browder's Domaln Invartance Theorem [1], $T\left(R^{n}\right)$ is an open subset of $R^{n}$. From Lemma 1 we have that $T\left(R^{n}\right)$ is a closed subset of $R^{n}$, hence $T\left(R^{n}\right)=R^{n}$ and thus $R^{n} \in \mathbb{E}$. $C^{n}$ 1s lsometris to $R^{2 n}$ therefore, $C^{n} \in E$. Since each fintte dimenstonal Banach space $1 \mathrm{~s}$ 1somorphic to $\mathrm{R}^{\mathrm{n}}$ or $\mathrm{C}^{\mathrm{n}}$ for some $\mathrm{n}$, thus each finite dimenstonal Banach space is an element of $Z$.

To find a class of Banach spaces that has no elements in $\mathbf{E}$, we can 
use the following lenma.

Lemma 2. If $x$ is a Banach space, then the following statements are equivalent:

(1) $\mathbf{X} \in \mathbf{E}$.

(11) If $T: X+X$ where $T$ is a contlnuous and expanding map, then $\theta \in T(X)$.

(111) If $\mathrm{T}: \mathrm{X} \rightarrow \mathrm{X}$ where $\mathrm{T}$ is a continuous and expanding map, then $T(X)=X$.

Proof. It is clear that (111) implies (1). To see that (1) Imp11es (11), suppose $X \in E, F: X \rightarrow X$ where $F$ is a continuous and expanding map. There exists $h>1$ such that $F \in E(h)$. Deftne $T: x \rightarrow x$ by $T(x)=F^{n}(x)+x$, where $n \in z^{+}$such that $h^{n}>2$. Since $\|T(x)-T(y)\| \geq h^{n_{x}}-y\|-\| x-y \|$, we have that $T$ 1s a continuous and expanding map. Thus, there extsts $p \in X$ such that $p=T(p)=F^{n}(p)+p$ and hence $\theta \in F(X)$.

To see that (11) implies (111), suppose $F: X+X, F$ is a continuous and expanding map and $x_{0} \in X$. Define $T: X+X$ by $T(x)=F(x)-x_{0} \cdot T$ Is a continuous and expanding map, thus there exlsts $p \in X$ auch that $\theta=F(p)-x_{0}$ and hence $F(X)=X$.

Theorem 4. Let $X$ denote a Banach apace. If there exists $T: X+X$ where $T$ is a linear homeomorphism and $T(X) \neq X$, then $X \notin E$.

Proof. Suppose $T$ is a 11near homeomorphism that maps $X$ into $X$ where $T(X) \neq X$. Define $F: X+X$ by $F(x)=2\left\|T^{-1}\right\| T(x) . \quad F \quad$ is a continuous and expanding map and $F(X)=T(X) \neq X$, thus by temma $2, X \notin X$ 
REFERENCE

1. James Dugundf1, Topology, A11yn and Bacon, Inc., 1966. 